\title{
Unified Control Strategy of PV Inverter Mixed Power Quality Control*
}

\author{
Jie Zhang, Kai Yao, Liang Li, Wei Zhao \\ School of Electrical \& Electronic Engineering, HuBei University of Technology, Wuhan, China \\ Email: haolihai2010@163.com \\ Received April, 2013
}

\begin{abstract}
Due to the restriction of light illumination condition, the effective utilization of PV grid-connected systems is very low. In view of this question, this paper presents a unified control strategy based on PV grid-connected and active power filter. The distributed small-capacity grid-connected inverter is chose to be the research object. It is preferential to eliminate the deviation between power qualities of the national standards according to the control of decision tables, and adjust the proportion of the compensation currents dynamically. Finally, the simulation results demonstrate the effectiveness of this unified control strategy.
\end{abstract}

Keywords: PV Inverter; APF; Unified Control; Decision Table

\section{Introduction}

Due to the alternation of days-nights and the change of the weather, the effective utilization of photovoltaic gridconnected system is very low, year time equals approximately to the annual sunshine hours. Meanwhile, the pollution of reactive power and harmonic to the grid is growing up so fast with the wide application of power electronic devices in the distribution system.

Based on the situations above, researchers at home and abroad are trying to make the inverter realizes the function of active power filtering based on PV grid-connected system in recent years. In [1, 2], they have realized the two kinds of functions above at the identical inverter, but the system works in the model of PV grid-connected when it is sunny, or in the model of active power filtering. This way of switching automatically between two models cannot take full advantage of the capacity of the inverter. In [3-8], part or all of the spare capacity of the inverter has been taken into account to compensate reactive power or harmonic. But the priority and the proportional allocation problem of the compensation currents are not considered, cannot achieve the optimal compensation for the load dynamically and instantaneously.

For the problems above, this article advances a novel method to unified control the PV grid-connected system and APF. Put the technologies of them together through the overall arrangement of the decision table. Make it have the functions of PV grid-connected and improving the power quality.

\footnotetext{
*This work was supported in part by Natural fund key projects in Hubei Province, China. (2010CDA018)
}

\section{System Structure \& Control Principle}

It is feasible to combine power quality optimization and PV grid-connected technology together to implement unified control for them from the analysis above. The structure of the unified control system is shown in Figure 1.

The control principle of the unified control system is summarized as follows: the active, reactive and harmonic currents are put into the decision table after they are detected and separated from the load current. Then the given currents which are output from the decision table compare with the grid-connected currents to produce PWM which controls the turn-on and turn-off of the power electronic devices. So the unified control system can realize electricity generation, harmonic suppression and reactive power compensation at the same time.

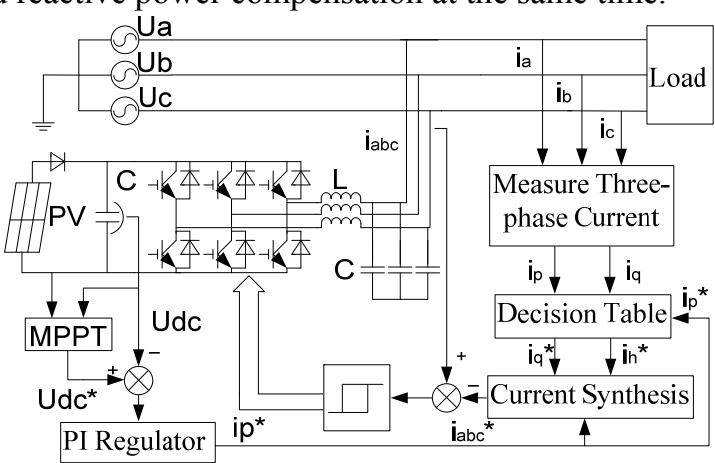

Figure 1. The structure of the unified control system.

In the control process above, extraction and separation of the currents is the key to complete the active power 
filtering. This article uses ip-iq method to realize the separation of active, reactive and harmonic currents, the principle is shown in Figure 2.

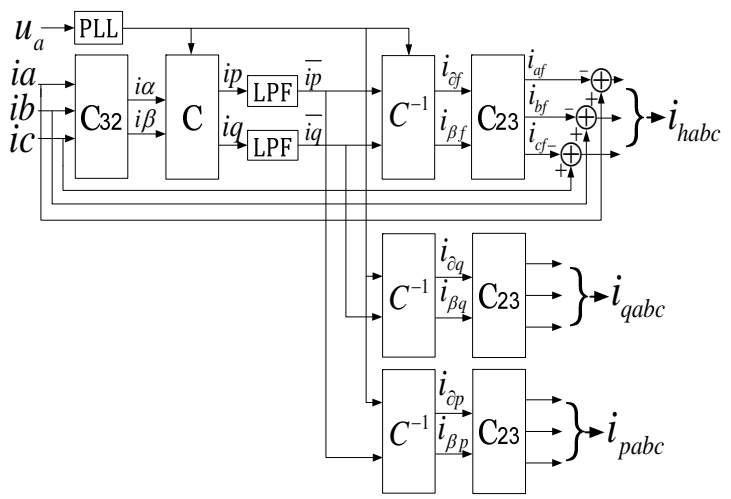

Figure 2. The detection of active, reactive and harmonic currents.

\section{Decision Table Control}

The power factor $\cos \varphi$ and harmonic coefficient THD of the grid can be calculated from the data above.

$$
\cos \varphi=\frac{I_{\mathrm{p}}}{\sqrt{{I_{\mathrm{p}}^{2}+I_{\mathrm{q}}^{2}}^{2}}}, T H D=\frac{\operatorname{RMS}\left(I_{h}\right)}{\operatorname{RMS}\left(I_{f}\right)}
$$

Dual closed-loop vector control is the control of this system based on which the outer-loop voltage to maintain the stability of the DC side voltage.

The active instruction current is calculated as follows:

$$
i_{p}^{*}=\Delta i_{c p}-i_{p v}
$$

In this system, the decision table is the core which adjusts the proportion of reactive and harmonic compensation currents dynamically. The unified control system outputs compensation currents selectively accordance with the priority to eliminate the larger side which deviates from the national standards, then combines with the capacity restriction of the grid-connected inverter. The decision processes of the decision table are summarized as follows:

1) When $T H D \leq 5 \% \& \cos \varphi \geq 0.9$. No control quantity is output from the decision table, the inverter just outputs active current.

2) THD $>5 \% \& \cos \varphi \geq 0.9$. The total deviation of the harmonic current will be compensated if the remaining capacity of the inverter is large enough. Otherwise, the inverter will keep working under full power state to compensate harmonic current.

3) $\mathrm{THD} \leq 5 \% \& \cos \varphi<0.9$. The control idea is the same as compensating harmonic.

4) THD $>5 \% \& \cos \varphi<0.9$. The deviation of the reactive and harmonic between the national standards will be considered, and the larger deviation will be compensated first. If there is capacity left after the larger deviation has been compensated totally, then the left capacity of the inverter will be used to compensate the other deviation. Or the inverter only compensates the larger deviation if the remaining capacity of the inverter is too little.

The specific control flow of the decision table is shown in Figure 3.

In the figure above, $\Delta i_{h}, \Delta i_{q}$ and expressions are shown respectively as follows:

$\Delta \mathrm{i}_{\mathrm{h}}=\mid$ THD $-0.05\left|* \sqrt{\mathrm{i}_{\mathrm{p}}{ }^{2}+\mathrm{i}_{\mathrm{q}}{ }^{2}}, \quad \Delta \mathrm{i}_{\mathrm{q}}=\right| \frac{\sqrt{1-\cos \varphi^{2}}}{\cos \varphi}-\frac{\sqrt{1-0.9^{2}}}{0.9} \mid * \mathrm{i}_{\mathrm{p}}$

Expression 1: $i_{h}^{*}=0, i_{q}^{*}=0$;

Expression 2: $i_{h}{ }^{*}=i_{\max }-i_{p} *, i_{q}^{*}=0$;

Expression 3: $i_{h}{ }^{*}=|T H D-0.05| * \sqrt{i_{p}{ }^{2}+i_{q}{ }^{2}}, i_{q}{ }^{*}=0$;

Expression 4: $i_{h} *=|T H D-0.05| * \sqrt{i_{p}{ }^{2}+i_{q}{ }^{2}}$,

$$
i_{q}^{*}=\left|\frac{\sqrt{1-\cos \varphi^{2}}}{\cos \varphi}-\frac{\sqrt{1-0.9^{2}}}{0.9}\right| * i_{p} ;
$$

Expression 5: $i_{h}{ }^{*}=|T H D-0.05| * \sqrt{i_{p}{ }^{2}+i_{q}^{2}}$,

$$
i_{q}^{*}=\sqrt{\left(i_{\max }-\Delta i_{h}\right)^{2}-i_{p}{ }^{2}} ;
$$

Expression 6: $i_{q} *=\left|\frac{\sqrt{1-\cos \varphi^{2}}}{\cos \varphi}-\frac{\sqrt{1-0.9^{2}}}{0.9}\right| * i_{p}$,

$$
i_{h}^{*}=i_{\max }-\sqrt{i_{p}^{2}+\Delta i_{q}^{2}}
$$

Expression 7: $i_{q}{ }^{*}=i_{\max }-i_{p} *, \quad i_{h}{ }^{*}=0 ;$

Expression 8: $i_{q}{ }^{*}=\left|\frac{\sqrt{1-\cos \varphi^{2}}}{\cos \varphi}-\frac{\sqrt{1-0.9^{2}}}{0.9}\right| * i_{p}$,

$$
i_{h}^{*}=0 \text {. }
$$

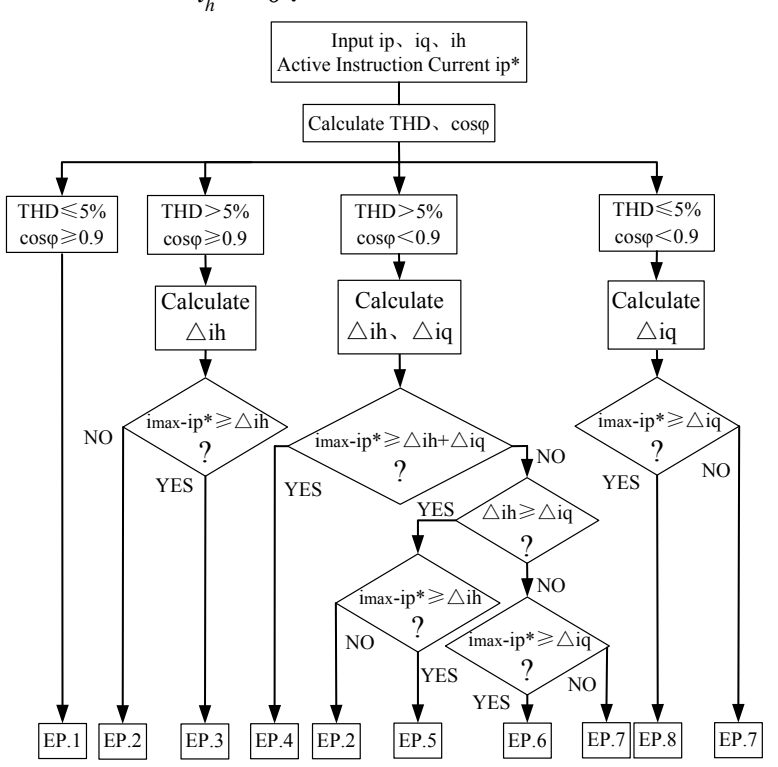

Figure 3. The decision table control process.

In this article, the THD and $\cos \varphi$ are calculated to generate the given currents $i_{p a b c} *, i_{q a b c} *$ and $i_{h a b c} *$ 
through the decision table and current amplitude unitized, the synthesis algorithm principle is shown in Figure 4.

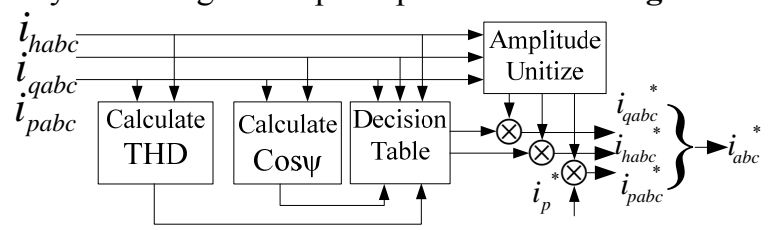

Figure 4. The synthesis algorithm of the given current.

The given currents $i_{p a b c} *, i_{q a b c} *$ and $i_{h a b c} *$ are superimposed to $i_{a b c} *$, then the PWM waves are generated to control the inverters how to work by a hysteresis comparison between above-mentioned $i_{a b c} *$ and gridconnected current $i_{a b c}$. So the system can achieve functions of both PV grid-connected power generation and active power filtering simultaneously.

\section{Simulation and Analysis}

In order to verify the effectiveness of this control method, the system simulation model is built in the environment of MATLAB/simulink according to the Figure 1. The line voltage of the power is $380 \mathrm{VAC} / 50 \mathrm{~Hz}$, the given voltage of DC side is $700 \mathrm{~V}$, the load is uncontrollable three-phase rectifier bridge which is resistive and inductive, and the rated capacity of the inverter is 10 kVA.

In order to facilitate the comparison and analysis of the results, the loads are the same in the simulation 1) and 2) below.

1) When the system improves the power quality first and then PV grid-connected, the simulation result is shown in Figure 5. At $0.05 \mathrm{~s}$, the system begins to compensate reactive and harmonic currents. At $0.11 \mathrm{~s}$, the system outputs active current to the grid as soon as the switch closes. As can be seen from the figure below, the THD of the grid is reduced to $3.23 \%$ from $21.69 \%$ and the $\cos \varphi$ is increased to 0.91 from 0.86 . Because the active current is fed into the grid at $0.11 \mathrm{~s}$, the active current provided by the grid is reduced to $13.2 \mathrm{~A}$ from $27.2 \mathrm{~A}$.

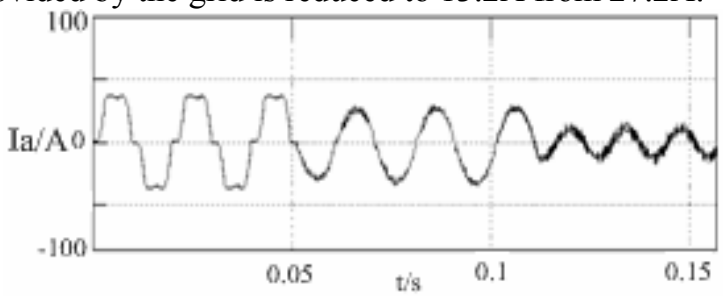

Figure 5. A phase current waveform of the grid.

These results indicate that the unified control system has the dual functions of PV grid-connected and active power filtering. The effects are satisfactory.

2) When the system compensates reactive power, harmonic and the both of them respectively, the simulation results are shown from (1) to (3) in Figure 6.

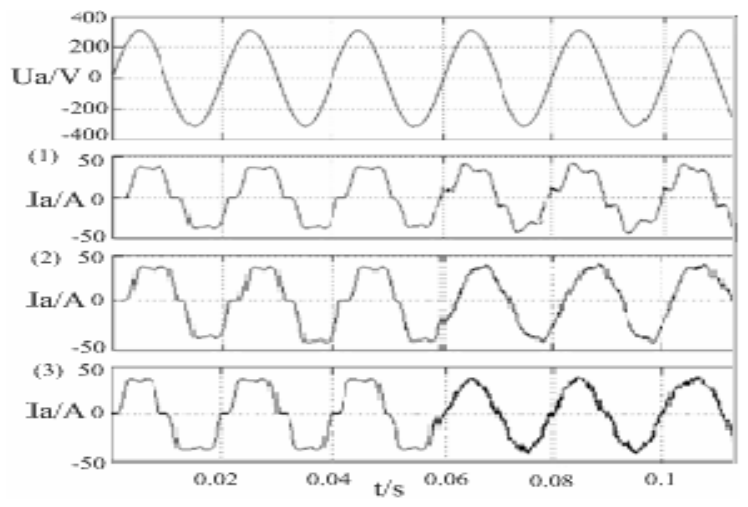

Figure 6. A phase current waves with different compensation methods.

Table 1. The and $\cos \psi$ of the grid (when the system compensates different currents ).

\begin{tabular}{lcccc}
\hline \multirow{2}{*}{$\begin{array}{c}\text { The Quantity of } \\
\text { Compensation }\end{array}$} & \multicolumn{2}{c}{ Initial State THD $\cos \varphi$} & Compensation THD $\cos \varphi$ \\
\cline { 2 - 5 } & \multicolumn{2}{c}{$(0 \sim 0.04 \mathrm{~s})$} & \multicolumn{2}{c}{$(0.05 \mathrm{~s} \sim 0.3 \mathrm{~s})$} \\
\hline Reactive Power & $21.69 \%$ & 0.86 & $21.69 \%$ & 0.97 \\
Harmonic & $21.69 \%$ & 0.86 & $2.23 \%$ & 0.86 \\
Both & $21.69 \%$ & 0.86 & $2.23 \%$ & 0.97 \\
\hline
\end{tabular}

As can be seen from the data in Table 1, the THD is reduced significantly from $21.69 \%$ to $2.23 \%$, and the $\cos \varphi$ is increased from 0.86 to 0.97 , the effects expected have been achieved.

3) The loads are changing ceaselessly all the time, the difference of the loads determines whether the inverter has sufficient capacity to compensate reactive power and harmonic, also determines the different control method.

The simulation results are shown in Figure 7 and the corresponding THD and $\cos \varphi$ are shown in Table 2 when the loads are changing.

As can be seen from Figure 7 and Table 2:

When the load is light, the capacity of the inverter is large enough to be used to compensate almost all of the reactive power and harmonic. Otherwise, only a part of them can be compensated.

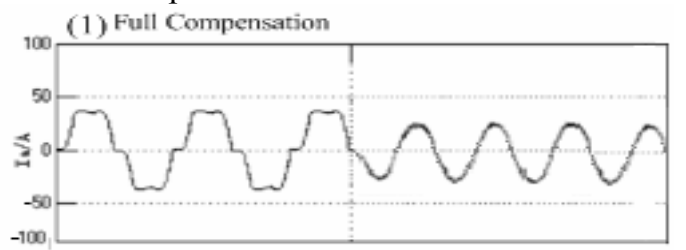

(2) Part Compensation

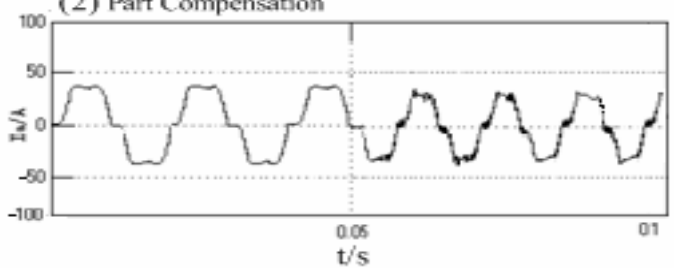

Figure 7. A phase current waves with different loads. 
Table 2. The and cos $\psi$ of the grid (when the load is changing).

\begin{tabular}{|c|c|c|c|c|}
\hline \multirow{3}{*}{$\begin{array}{l}\text { Load (light, heavy) } \\
\qquad P_{\max } \geqslant P_{\mathrm{q}}+P_{h}\end{array}$} & \multicolumn{2}{|c|}{ Initial State THD $\cos \varphi$} & \multicolumn{2}{|c|}{ Compensation THD $\cos \varphi$} \\
\hline & \multicolumn{2}{|c|}{$(0 \sim 0.04 \mathrm{~s})$} & \multicolumn{2}{|c|}{$(0.05 \mathrm{~s} \sim 0.3 \mathrm{~s})$} \\
\hline & $21.69 \%$ & 0.86 & $2.23 \%$ & 0.97 \\
\hline$P_{\max }<P_{q}+P_{h}$ & $33.44 \%$ & 0.62 & $17.64 \%$ & 0.88 \\
\hline
\end{tabular}

\section{Conclusions}

This paper presents a unified control strategy of PV inverter mixed power quality control, which adjusts the size and conjunction of compensation currents accurately and flexibly according to the changes of the load and the grid. So the capacity of the PV grid-connected system can be made full use.

Through this novel unified control system, not only can improve the utilization of PV grid-connected system, but also provide a viable idea and a beneficial attempt to the power quality control of the distributed PV system.

\section{REFERENCES}

[1] H. L. Wang, J. W. Su, G. R. Zhang, et al., "With Reactive Power Compensation and Harmonic Suppression of ReSearch on PV Grid-connected Power Conditioner Control," Acta Energiae Solaris Sinica (monthly), Vol. 27, No. 6, 2006, pp. 540-545.
[2] P. Wang and C. B. Zheng, "Harmonic Suppression and Reactive Power Compensation of Grid-Connected PV Systems," Microcomputer and application, Vol. 30, No. 14, 2011, pp. 58-61.

[3] L. B. Wu, Z. M. Zhao, J. Z. Liu, et al., "Single-stage Three-phase Pv Grid System with Reactive Power Compensation," Transactions of China Electrotechnical Society, Vol. 21, No. 1, 2006, pp. 28-32.

[4] H. L. Wang, J. W. Su, G. R. Zhang, et al., "With Reactive Power Compensation and Harmonic Suppression of Research on Photovoltaic Grid-connected Power Conditioner Control," Acta Energiae Solaris Sinica (monthly), Vol. 27, No. 6, 2006, pp. 540-545.

[5] P. Wang and C. B. Zheng, "Harmonic Suppression and Reactive Power Compensation of Grid-connected PV Systems," Microcomputer and Application, Vol. 30, No. 14, 2011, pp. 58-61.

[6] L. B. Wu, Z. M. Zhao, J. Z. Liu, et al., "Single-stage Three-phase Photovoltaic Grid System with Reactive Power Compensation," Transactions of China Electrotechnical Society, Vol. 21, No. 1, 2006, pp. 28-32.

[7] C. H. Wu, J. M. Wang, W. M. Chen, et al., "Unified Control of the Single-phase Photovoltaic Grid with Active Filter," Transactions of China Electrotechnical Society, Vol. 26, No.10, 2011, pp. 103-109.

[8] H. Jin and X. X. Liang, "With Function of Shunt Active Power Filter Based on Photovoltaic Grid-connected Power Systems Research,” Ship Power Technology, Vol. 31, No. 5, 2011, pp. 4-7. 